\title{
Orbital and physical parameters of eclipsing binaries from the All-Sky Automated Survey catalogue
}

\section{AK Fornacis: a rare, bright K-type eclipsing binary ${ }^{\star}$}

\author{
K. G. Hełminiak ${ }^{1,2,3}$, R. Brahm ${ }^{1}$, M. Ratajczak ${ }^{2}$, N. Espinoza ${ }^{1}$, A. Jordán ${ }^{4,1}$, M. Konacki ${ }^{2,5}$, and M. Rabus ${ }^{1,6}$ \\ ${ }^{1}$ Instituto de Astrofísica, Pontificia Universidad Católica de Chile, Av. Vicuña Mackenna 4860, 7820436 Macul, Santiago, Chile \\ e-mail: [xysiek;rbrahm;nsespino;ajordan;mrabus]@astro.puc.cl \\ 2 Nicolaus Copernicus Astronomical Center, Department of Astrophysics, ul. Rabiańska 8, 87-100 Toruń, Poland \\ e-mail: [xysiek;milena;maciej]@ncac.torun.pl \\ 3 Subaru Telescope, National Astronomical Observatory of Japan, 650 North Aohoku Place, Hilo HI 96720, USA \\ 4 Millennium Institute of Astrophysics, Av. Vicuña Mackenna 4860, 7820436 Macul, Santiago, Chile \\ 5 Astronomical Observatory, A. Mickiewicz University, ul. Słoneczna 36, 60-286 Poznań, Poland \\ ${ }^{6}$ Max-Planck-Institut für Astronomie, Königstuhl 17, 69117 Heidelberg, Germany \\ Received 21 December 2012 / Accepted 13 May 2014
}

\section{ABSTRACT}

\begin{abstract}
Aims. We present the results of the combined photometric and spectroscopic analysis of a bright $(V=9.14)$, close $(d=31$ pc $)$, late-type detached eclipsing binary, AK Fornacis. This $P=3.981 \mathrm{~d}$ system has not been previously recognised as a double-lined spectroscopic binary, and this is the first full physical model of this unique target.

Methods. With the FEROS, CORALIE, and HARPS spectrographs, we collected a number of high-resolution spectra to calculate radial velocities of both components of the binary. Measurements were done with our own disentangling procedure and the TODCOR technique, and were later combined with the photometry from the ASAS and SuperWASP archives. We also performed an atmospheric analysis of the component spectra with the Spectroscopy Made Easy package.

Results. Our analysis shows that AK For consists of two active, cool dwarfs having masses of $M_{1}=0.6958 \pm 0.0010$ and $M_{2}=$ $0.6355 \pm 0.0007 M_{\odot}$ and radii of $R_{1}=0.687 \pm 0.020$ and $R_{2}=0.609 \pm 0.016 R_{\odot}$, respectively, which are slightly less metal-abundant than the Sun. Parameters of both components are well reproduced by the models.

Conclusions. AK For is the brightest system among the known eclipsing binaries with K- or M-type stars. Its orbital period is one of the longest, and its rotational velocities is one of the lowest, which allows us to obtain very precise radial velocity measurements. The precision in physical parameters that we obtained places AK For among the binaries with the best mass measurements in the literature. It also fills the gap in our knowledge of stars in the range of $0.5-0.8 \mathrm{M}_{\odot}$ and between short and long-period systems. All this makes AK For a unique benchmark for understanding the properties of low-mass stars.
\end{abstract}

Key words. binaries: eclipsing - binaries: spectroscopic - stars: fundamental parameters - stars: individual: AK Fornacis

\section{Introduction}

During the past few years we have witnessed a remarkable improvement in the study of lower main-sequence stars - mainly by the increasing number of eclipsing binaries found to contain K- and M-type dwarfs. The interest in those objects has increased recently with the new exoplanet search surveys that are dedicated especially to them, such as the WFCAM Transit Survey ${ }^{1}$. The knowledge of the host star parameters, especially mass and radius, is necessary to constrain the characteristics of the planet (Demory et al. 2011). Another justification for studying late-type stars are the still unexplained discrepancies between the models and observations. We still do not fully understand why in most cases, sometimes surprisingly, the stars are larger and cooler than predicted (e.g. Lacy 1977; Popper 1997; Torres \& Ribas 2002; Morales et al. 2009; Irwin et al. 2011), while the same characteristics are nicely reproduced in

* Based on observations collected at the European Southern Observatory, Chile under programmes 088.D-0080, 089.D-0097, 089.C-0415, 090.C-0280 and 090.D-0061 and through CNTAC proposals CN-2012A-21 and CN-2013A-93.

1 http://www.ast.cam.ac.uk/ sth/wts/index.html others (Thompson et al. 2010; Feiden et al. 2011; Hełminiak \& Konacki 2011). Unfortunately, most newly discovered systems tend to be faint and have large rotational velocities due to short periods $(P<3 \mathrm{~d})$; thus, their properties are difficult to measure accurately. Only a handful of them have their masses and radii measured with an accuracy better than 3\% (with systematics taken into account properly), thus allowing for meaningful tests of the stellar models (Torres et al. 2010). Each new system is highly valuable, and in this paper we present the analysis of the brightest one known to date, which also has one of the most accurate mass measurements among the eclipsing binaries until now.

The variable nature of AK Fornacis (HD 21703, HIP 16247, ASAS J032923-2406.1; hereafter AK For) was discovered by the HIPPARCos mission, and the star was classified as probably eclipsing in the 74th Special Name-list of Variable Stars (Kazarovets et al. 1999). Before that it was only known as a K-type star (Upgren et al. 1972) and an X-ray source (Gioia et al. 1990). Otero (2003) gave the first period estimation, $3.981 \mathrm{~d}$, on the basis of HIPPARCos and the All-Sky Automated Survey (ASAS; Pojmański 2002) data available at that time; however, AK For is not listed in the ASAS Catalogue of Variable Stars. 
Table 1. Literature and catalogue data for AK For.

\begin{tabular}{lcc}
\hline \hline Parameter & Value & Ref. \\
\hline$\alpha_{\text {ICRS }}$ & $03: 29: 22.87422$ & 1 \\
$\delta_{\text {ICRS }}$ & $-24: 06: 03.0926$ & 1 \\
$\pi[\mathrm{mas}]$ & $32.19(1.13)$ & 1 \\
$\mu_{\alpha}[\mathrm{mas} / \mathrm{yr}]$ & $220.58(95)$ & 1 \\
$\mu_{\delta}[\mathrm{mas} / \mathrm{yr}]$ & $99.53(1.66)$ & 1 \\
$B$ [mag] & 10.46 & 2 \\
$V_{\text {Mer }}[\mathrm{mag}]$ & 9.36 & 2 \\
$V_{\text {ASAS }}[\mathrm{mag}]$ & 9.135 & 3 \\
$J[\mathrm{mag}]$ & $7.031(21)$ & 4 \\
$H$ [mag] & $6.510(53)$ & 4 \\
$K$ [mag] & $6.262(33)$ & 4 \\
Sp. type & K3 V k Fe+0.4 & 5 \\
$P_{\text {orb }}[\mathrm{d}]$ & 3.981 & 6 \\
\hline
\end{tabular}

References. 1: HIPPARCos (van Leeuwen 2007); 2: Mermilliod (1986); 3: our fit to ASAS data; 4: 2MASS (Skrutskie et al. 2006); 5: Gray et al. (2006); 6: Otero (2003).

The only spectroscopic study was done in low resolution by Gray et al. (2006), who classified AK For as a K3 V, "chromospherically very active" star. Finally, Bailer-Jones (2011) estimated the effective temperature and extinction towards the star on the basis of multiband photometry, but his method is not suitable for binaries, so both values are overestimated ( $5350 \mathrm{~K}, 1.28 \mathrm{mag})$. The summary of catalogue and literature data is presented in Table 1 . Note the discrepancy between ASAS and Mermilliod's (1986) values of the $V$ magnitude. The system's $T_{\text {eff }}$ calculated from $V-K$ colours and calibrations by Worthey \& Lee (2011) is $\sim 4100 \mathrm{~K}$ for Mermilliod (1986) and $\sim 4350 \mathrm{~K}$ for ASAS.

\section{Observations}

\subsection{Spectroscopy}

With the Fiber-fed Extended Range Optical Spectrograph (FEROS; Kaufer et al. 1999) we collected twelve high-resolution $(R \sim 40000)$ spectra in November 2011, September 2012, and March 2013. They were supplemented with six $R \sim 70000$ spectra taken in February 2013 with the CORALIE spectrograph, which is attached to the 1.2-m Euler Telescope in La Silla and two $R \sim 115000$ spectra from the High Accuracy Radial velocity Planet Searcher (HARPS; Mayor et al. 2003) that were obtained in quadrature on September 09, 2012. The FEROS and CORALIE data were reduced with a dedicated Python-based pipeline (for a description see: Jordán et al. 2014), which was initially built for CORALIE data reduction, but was modified to deal with FEROS data. The HARPS spectra were reduced onsite with the available Data Reduction Software (DRS).

\subsection{Photometry}

The $V$-band photometry of AK For, which is publicly available from the ASAS Catalogue ${ }^{2}$, spans from November 2000 to December 2009 and contains 883 good quality points (flagged "A" in the original data). The time span of the public Wide-Angle Search for Planets (SuperWASP; Pollacco et al. 2006) data ${ }^{3}$ is much shorter - from August 2006 till February 2010 - but with

\footnotetext{
2 http://www.astrouw.edu.pl/asas/?page=aasc

3 http://exoplanetarchive.ipac.caltech. edu/applications/ExoTables/search.html? dataset=superwasptimeseries
}

only a few, practically useless points after Feb. 2008. Due to the orbital period very close to four days, the eclipses are not always observable in the night, so we initially selected only 2007-2008 data in the case of SuperWASP, where eclipses were visible. We later limited ourselves to 2709 data points with individual errors that are smaller than 0.03 mag. This threshold level was a result of the further analysis, in which we wanted to keep as many good quality data points as possible (especially in eclipses), while minimizing the systematics in the resulting radii at the same time. We also noted that the depths of single minima vary slightly, but it was impossible to conclude if it is a real effect (due to spots for example) or an uncorrected systematic. The AK For is known to be an active system but no consistent out-of-eclipse modulation was found in the SuperWASP photometry.

To check for the spot-originated brightness variations, we performed $V$-band observations with the PISCO Telescope, which is attached to the main tube of the 1.2-m Euler Telescope in La Silla. The PISCO is a $31.75 \mathrm{~cm}$ (12.5 in) aperture reflector by RC optical systems with a Finger Lakes Instrumentation (FLI) Proline-09000 camera, which is equipped with a $3056 \times$ 3056 pixel Kodak KAF-09000 CCD. With the pixel scale of $0.862^{\prime \prime} /$ pix, the field of view is $44^{\prime \prime} \times 44^{\prime \prime}$ and strongly vignetted in the corners. It contains a set of standard JohnsonCousins $U, B, V, R$, abnd $I$ filters, which are supplemented by near-IR $Z$ and $Y$. It works with the CORALIE spectrograph, allows for simultaneous photometry and spectroscopy and takes exposures while CORALIE's shutter is open. With PISCO we secured 58 images in the $V$ band with exposure times of $18 \mathrm{~s}$. They were taken exactly in the same time as CORALIE spectra from the nights of February 24, 25 and 26, 2013. The standard CCD reduction steps, which utilize bias and sky-flat images, were performed in IRAF, and simple aperture photometry under the task apphot. The target was by far the brightest star in the field, so the selection of comparison stars was difficult. We inspected seven other stars and found two that did not show any significant brightness variations but, unfortunately, are about 2 mag fainter than AK For. We only wanted to perform a relative photometry, to detect any variability, so we did not transform PISCO magnitudes to the standard Johnson's system, nor did we observe photometric standard stars.

\section{Analysis}

\subsection{Radial velocities and the orbital solution}

Radial velocities (RVs) were initially calculated with our implementation of the two-dimensional cross-correlation technique (TODCOR; Zucker \& Mazeh 1994) with synthetic spectra that were used as templates. Due to the poor signal-to-noise ratio $(\mathrm{S} / \mathrm{N})$ in the blue part, the limits implemented to the CORALIE and FEROS reduction pipelines (optimized for high-precision $\mathrm{RVs}$ ) and the limitations of the synthetic templates the following wavelength ranges were used: 4135-6500 $\AA$ for FEROS, 4400-6500 $\AA$ for CORALIE, and 3800-6500 $\AA$ for HARPS. One of the TODCOR's features is that it also gives the most probable flux ratio of the two components. We found it to be 0.423 and that it is pretty stable along the $V$ and SuperWASP bands. Later, we used the FEROS spectra to perform disentangling, as described in Konacki et al. (2010), and to obtain separate spectra of the two components. We used them to perform the spectral analysis and for final RV measurements. For the FEROS data, we followed the procedure of least-squares fitting, as described in Konacki et al. (2010), and for CORALIE and HARPS, we used the disentangled spectra as templates in TODCOR. 
K. G. Hełminiak et al.: Orbital and physical parameters of eclipsing binaries from the ASAS catalogue. VI.

Table 2. Individual RV measurements, errors, and residuals (all in $\mathrm{km} \mathrm{s}^{-1}$ ) of the components of AK For with the orbital phase, exposure times (in seconds) and $\mathrm{S} / \mathrm{N}$ of the spectra.

\begin{tabular}{lrrrrrrrrrr}
\hline \hline JD-2 450000 & $v_{1}$ & $\sigma_{1}$ & $(\mathrm{O}-\mathrm{C})_{1}$ & $v_{2}$ & $\sigma_{2}$ & $(\mathrm{O}-\mathrm{C})_{2}$ & Phase & $T_{\exp }$ & $S / N$ & Tel./Sp. \\
\hline 5876.681435 & -37.483 & 0.062 & -0.003 & 46.441 & 0.202 & -0.008 & 0.0965 & 450 & 90 & $2 \mathrm{~F}$ \\
5876.841349 & -50.637 & 0.049 & 0.031 & 60.928 & 0.155 & 0.026 & 0.1366 & 900 & 125 & $2 \mathrm{~F}$ \\
5877.644573 & -57.370 & 0.067 & -0.149 & 67.959 & 0.242 & -0.122 & 0.3384 & 750 & 120 & $2 \mathrm{~F}$ \\
5877.701489 & -53.645 & 0.061 & 0.003 & 64.000 & 0.231 & -0.165 & 0.3527 & 1200 & 90 & $2 \mathrm{~F}$ \\
5877.783808 & -47.668 & 0.038 & 0.018 & 57.725 & 0.139 & 0.092 & 0.3734 & 1200 & 95 & $2 \mathrm{~F}$ \\
5878.673272 & 42.913 & 0.056 & -0.001 & -41.535 & 0.185 & 0.043 & 0.5968 & 600 & 95 & $2 \mathrm{~F}$ \\
5878.759453 & 50.399 & 0.043 & 0.010 & -49.798 & 0.126 & -0.041 & 0.6184 & 450 & 90 & $2 \mathrm{~F}$ \\
5878.810493 & 54.428 & 0.039 & 0.021 & -54.178 & 0.137 & -0.024 & 0.6313 & 480 & 105 & $2 \mathrm{~F}$ \\
6179.782690 & -67.426 & 0.095 & 0.004 & 79.214 & 0.329 & -0.056 & 0.2336 & 900 & 85 & $3 \mathrm{H}$ \\
6179.879929 & -67.687 & 0.089 & 0.027 & 79.484 & 0.339 & -0.097 & 0.2580 & 780 & 90 & $3 \mathrm{H}$ \\
6195.817064 & -67.545 & 0.048 & 0.080 & 79.397 & 0.134 & -0.086 & 0.2613 & 480 & 120 & $2 \mathrm{~F}$ \\
6345.559577 & 52.316 & 0.054 & 0.094 & -51.594 & 0.136 & 0.075 & 0.8757 & 420 & 35 & $\mathrm{EC}$ \\
6346.511053 & -44.029 & 0.184 & -0.132 & 53.513 & 0.294 & -0.061 & 0.1147 & 600 & 25 & $\mathrm{EC}$ \\
6347.545693 & -47.323 & 0.060 & -0.001 & 57.305 & 0.144 & -0.021 & 0.3746 & 600 & 30 & $\mathrm{EC}$ \\
6348.542794 & 52.439 & 0.060 & -0.046 & -52.171 & 0.123 & -0.212 & 0.6251 & 480 & 30 & $\mathrm{ECp}$ \\
6349.537454 & 52.613 & 0.091 & 0.146 & -51.882 & 0.134 & 0.055 & 0.8749 & 420 & 25 & $\mathrm{ECp}$ \\
6350.536805 & -47.586 & 0.046 & -0.069 & 57.613 & 0.093 & 0.072 & 0.1259 & 600 & 30 & $\mathrm{ECp}$ \\
6376.509645 & 59.529 & 0.075 & -0.198 & -59.889 & 0.191 & 0.086 & 0.6502 & 600 & 90 & $2 \mathrm{~F}$ \\
6377.503283 & 44.095 & 0.062 & -0.054 & -42.758 & 0.173 & 0.172 & 0.8997 & 600 & 135 & $2 \mathrm{~F}$ \\
6383.474455 & -38.866 & 0.068 & -0.001 & 47.980 & 0.279 & 0.012 & 0.3997 & 600 & 75 & $2 \mathrm{~F}$ \\
\hline
\end{tabular}

Notes. The 2F marks the MPG 2.2-m/FEROS, the 3H the ESO 3.6-m/HARPS, and the EC the 1.2-m Euler/CORALIE data (with "p" meaning simultaneous observations with PISCO).

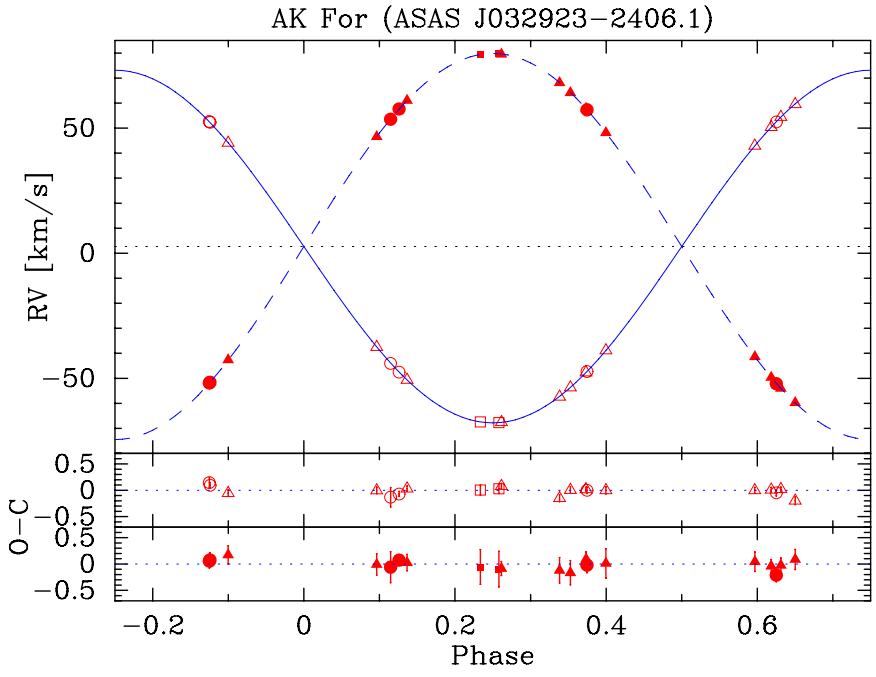

Fig. 1. Radial velocities and orbital solution for AK For. Solid line and empty symbols refer to the primary; dashed line and filled symbols to the secondary component. Triangles mark the FEROS, circles mark the CORALIE, and squares the HARPS data. The systemic velocity is marked with the dotted line. The residuals are plotted below with the (scaled) individual errors. The rms of the orbital fit is 82 and $96 \mathrm{~m} / \mathrm{s}$ for the primary and secondary, respectively.

The RV measurements were analysed with a simple procedure, which fits a double-keplerian orbit by using the LevenbergMarquartd algorithm. As free parameters, we set the orbital period $P$, the time of primary conjunction $T_{0}$, which corresponds to the primary (deeper) eclipse in this case, the velocity semiamplitudes $K_{1,2}$, the primary's systemic velocity $\gamma_{1}$, the difference in components systemic velocities $\gamma_{2}-\gamma_{1}$, and the difference between FEROS and CORALIE zero-points $2 \mathrm{~F}-\mathrm{EC}$, as measured separately for each component. We assumed that the HARPS has the same zero-point as FEROS, as we did not have enough HARPS data to securely constrain this difference. The HARPS measurements do not significantly outlay from the

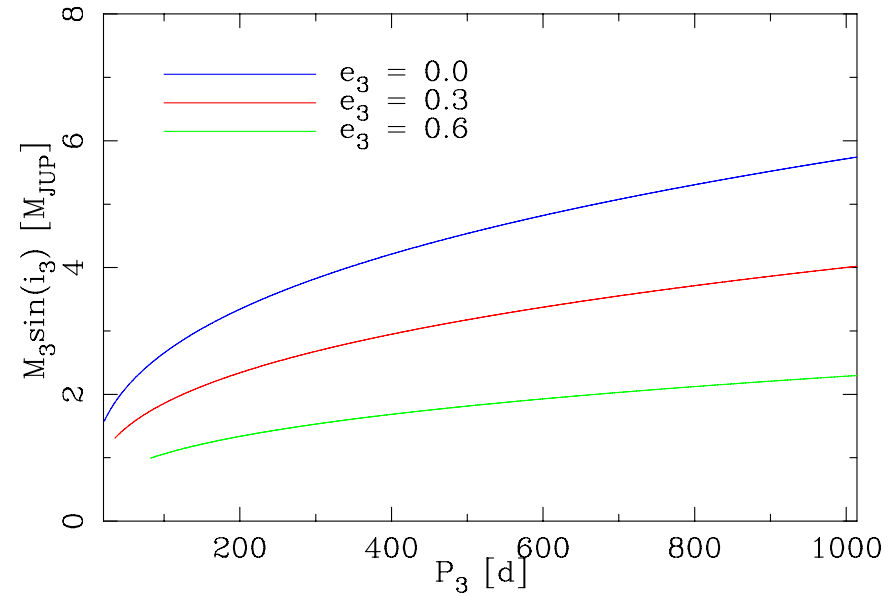

Fig. 2. Third body detection $M_{3} \sin \left(i_{3}\right)$ limits as estimated from our RV measurements for several values of outer orbit's eccentricities. We should be able to detect bodies with masses above these limits.

final solution, so we find this assumption correct. Initially, we also fitted for the eccentricity $e$ and the argument of the periastron $\omega$ but we did not find the orbit eccentric in this step or any other. Thus, we kept $e=0$ during the whole process. Finally, we ended up with 40 data points (20 for each component) and eight parameters.

To estimate reliable parameter uncertainties with systematics that are accounted for, we run 10000 bootstrap iterations. All the RV measurements with their uncertainties, residuals from the model RV curve, orbital phases, exposure times, and $\mathrm{S} / \mathrm{N}$ around $5500 \AA$ are shown in the Table 2 . The resulting orbital parameters are presented in Table 3 .

The precision of RVs that we have reached is good enough to look for massive circumbinary planets around the system. If we assume that we can detect any RV variation of the semiamplitude of at least $96 \mathrm{~m} / \mathrm{s}$, the rms of the secondary, we can calculate the mass of a body that could produce such a variation. We show those calculations in Fig. 2 as a function of the outer 


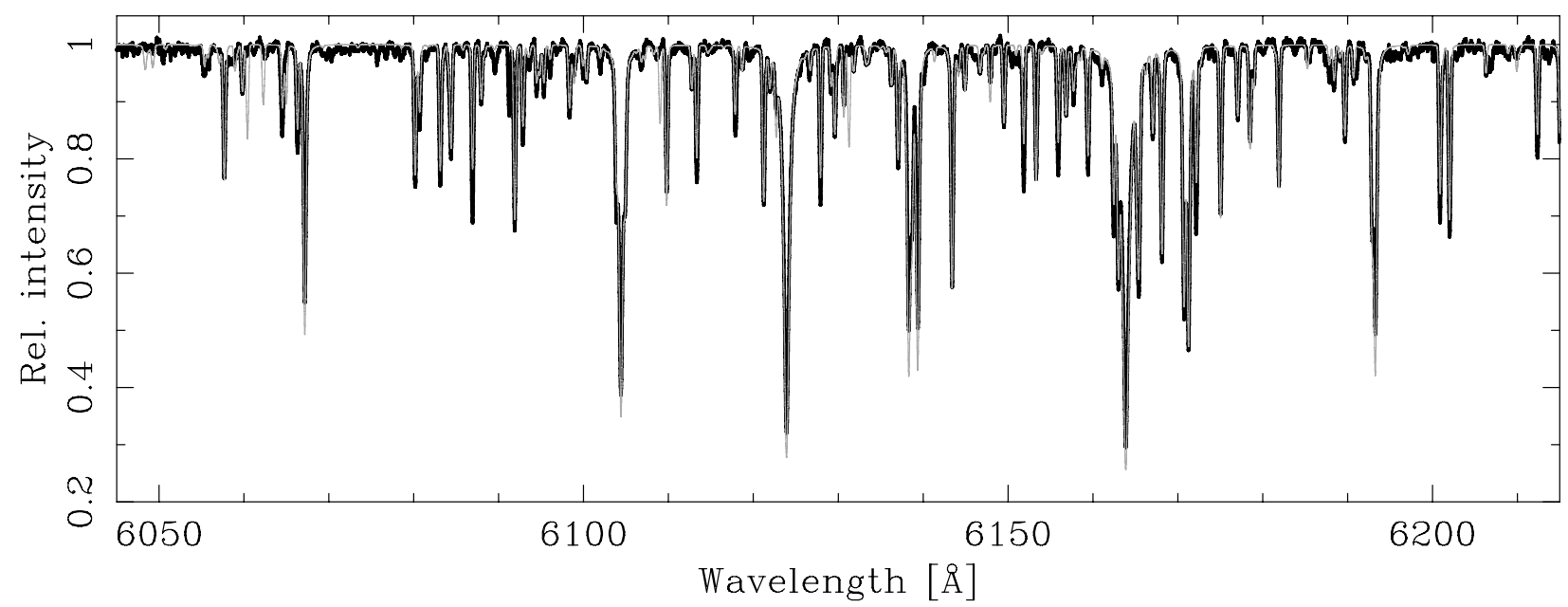

Fig. 3. Portion of the SME best fit (thin grey line) to the disentangled spectrum of the primary (thick black line).

Table 3. Orbital and physical parameters of AK For.

\begin{tabular}{|c|c|c|c|c|}
\hline \multirow{2}{*}{$\begin{array}{l}\text { AK For } \\
\text { Parameter }\end{array}$} & \multicolumn{2}{|c|}{ Primary } & \multicolumn{2}{|c|}{ Secondary } \\
\hline & Value & \pm & Value & \pm \\
\hline & \multicolumn{4}{|c|}{ Radial velocity fit } \\
\hline $\begin{array}{l}P[\mathrm{~d}] \\
T_{0}[\mathrm{JD}]\end{array}$ & \multicolumn{4}{|c|}{$3.9809913 \pm 0.0000045$} \\
\hline$K\left[\mathrm{~km} \mathrm{~s}^{-1}\right]$ & 70.471 & 0.031 & 77.136 & 0.051 \\
\hline$\gamma\left[\mathrm{km} \mathrm{s}^{-1}\right]$ & 2.667 & 0.028 & 2.516 & 0.115 \\
\hline$M \sin ^{3}(i)\left[M_{\odot}\right]$ & 0.6937 & 0.0010 & 0.6336 & 0.0008 \\
\hline $2 \mathrm{~F}-\mathrm{EC}\left[\mathrm{km} \mathrm{s}^{-1}\right]$ & -0.037 & 0.032 & 0.133 & 0.073 \\
\hline $2 \mathrm{~F}-3 \mathrm{H}\left[\mathrm{km} \mathrm{s}^{-1}\right]$ & 0.0 & - & 0.0 & - \\
\hline \multirow[t]{2}{*}{$\operatorname{rms}\left[\mathrm{km} \mathrm{s}^{-1}\right]$} & \multicolumn{2}{|c|}{0.082} & \multicolumn{2}{|c|}{0.096} \\
\hline & \multicolumn{4}{|c|}{ SME spectral analysis } \\
\hline$T_{\text {eff }}[\mathrm{K}]$ & 4690 & 100 & 4390 & 150 \\
\hline$[\mathrm{M} / \mathrm{H}]$ & -0.29 & 0.05 & -0.10 & -0.05 \\
\hline \multirow[t]{2}{*}{$v \sin (i)\left[\mathrm{km} \mathrm{s}^{-1}\right]$} & 9.52 & 0.22 & 8.27 & 0.42 \\
\hline & \multicolumn{4}{|c|}{ Light curve fit ${ }^{a}$} \\
\hline$i\left[{ }^{\circ}\right]$ & \multicolumn{4}{|c|}{$87.428 \pm 0.080$} \\
\hline$r[R / a]$ & 0.0591 & 0.0017 & 0.0524 & 0.0014 \\
\hline$J_{\mathrm{ASAS}}$ & \multirow{2}{*}{\multicolumn{4}{|c|}{$\begin{array}{l}0.528 \pm 0.028 \\
0.592 \pm 0.030\end{array}$}} \\
\hline$J_{\mathrm{SW}}$ & & & & \\
\hline & \multicolumn{4}{|c|}{ Adopted physical parameters } \\
\hline$a\left[R_{\odot}\right]$ & \multicolumn{4}{|c|}{$11.6318 \pm 0.0047$} \\
\hline$M\left[M_{\odot}\right]$ & 0.6958 & 0.0010 & 0.6355 & 0.0007 \\
\hline$R\left[R_{\odot}\right]$ & 0.687 & 0.020 & 0.609 & 0.016 \\
\hline $\log g$ & 4.607 & 0.025 & 4.673 & 0.023 \\
\hline$v_{\text {rot }}\left[\mathrm{km} \mathrm{s}^{-1}\right]^{b}$ & 8.72 & 0.25 & 7.73 & 0.21 \\
\hline $\log \left(L / L_{\odot}\right)$ & -0.687 & 0.045 & -0.908 & 0.064 \\
\hline \multirow[t]{2}{*}{$M_{\mathrm{bol}}[\mathrm{mag}]$} & 6.47 & 0.11 & 7.02 & 0.16 \\
\hline & \multicolumn{4}{|c|}{ From and based on HIPPARCOS } \\
\hline$d[\mathrm{pc}]$ & \multicolumn{4}{|c|}{$31.1 \pm 1.1$} \\
\hline \multirow[t]{2}{*}{$M_{V}[\mathrm{mag}]$} & 7.06 & 0.08 & 7.91 & 0.09 \\
\hline & \multicolumn{4}{|c|}{ From JKTABSDIM } \\
\hline$d[\mathrm{pc}]$ & \multicolumn{4}{|c|}{$32.4 \pm 1.6$} \\
\hline$M_{V}[\mathrm{mag}]$ & 6.91 & 0.17 & 7.68 & 0.27 \\
\hline
\end{tabular}

Notes. ${ }^{(a)}$ Combined ASAS and SuperWASP solutions. ${ }^{(b)}$ Assuming synchronization.

orbit's period and for three values of the outer orbit's eccentricity: $0.0,0.3$ and 0.6. The value of the $96 \mathrm{~m} / \mathrm{s}$ was assumed to be the RV semi-amplitude. The lower limit of the period is $21 \mathrm{~d}$ (the major semi-axis of $34.83 R_{\odot}$ ), which refers to the shortest stable circular orbit (Holman \& Wiegert 1999). For eccentric orbits, the limits are terminated at the shortest periods having the distance of periastron larger than $34.83 R_{\odot}$ (around 35 and $82 \mathrm{~d}$ ). The upper limit of the period is $1014 \mathrm{~d}$, which is twice the time span of our data. One can see that our radial velocities are precise enough to detect massive planets on stable orbits around AK For. If there was a third body orbiting within this range of periods, it must have the mass lower than the limit.

\subsection{Atmospheric parameters}

We performed a spectral atmospheric analysis using the Spectroscopy Made Easy package (hereafter SME; Valenti \& Piskunov 1996). The disentangled spectra were first scaled with the flux ratio obtained from TODCOR (0.423). We have also run SME with other flux ratios, but the resulting temperatures led to further inconsistencies in distances, for example.

Following the work of Valenti \& Fischer (2005) to obtain a continuum placement and derive more accurate parameters, we have chosen seven FEROS orders between 5317 and $6397 \AA$, where each of them was analysed separately. We used the list of atomic lines from the Vienna Atomic Line Database (VALD; Piskunov et al. 1995; Kupka et al. 1999), as generated for the Sun for the initial values described by Valenti \& Fischer (2005). We also adopted atmosphere models of Kurucz (1993). We set the $\log (g)$ for each component to the values obtained with JKTABSDIM (see: Table 3 and next section), the microand macro-turbulence velocities to 1 and $3 \mathrm{~km} \mathrm{~s}^{-1}$ respectively, while $T_{\mathrm{eff}},[\mathrm{M} / \mathrm{H}]$ and $v \sin (i)$ were fitted for every order. As starting values of temperatures, we used 4500 and $4150 \mathrm{~K}$ for the primary and secondary, respectively. For each order, we also started with three different values of $[\mathrm{M} / \mathrm{H}]:-1,-0.5$, and 0 . From all SME runs we took only those, for which the resulting $v \sin (i)$ was close to the value obtained from JKTEBOP (see next section). Of the $21 \mathrm{SME}$ runs for each component, only 13 and 9 was successful for the primary and secondary, respectively. Example of a successful fit to one of the orders of the primary's spectrum is presented in Fig. 3.

For the adopted values of effective temperatures, metallicities, and projected rotational velocities we took the average, and standard deviations as their uncertainties. Those values are given in Table 3 . The ratio of velocities agrees with the ratio of radii found in the light curve fitting (see next section), but the 
K. G. Hełminiak et al.: Orbital and physical parameters of eclipsing binaries from the ASAS catalogue. VI.

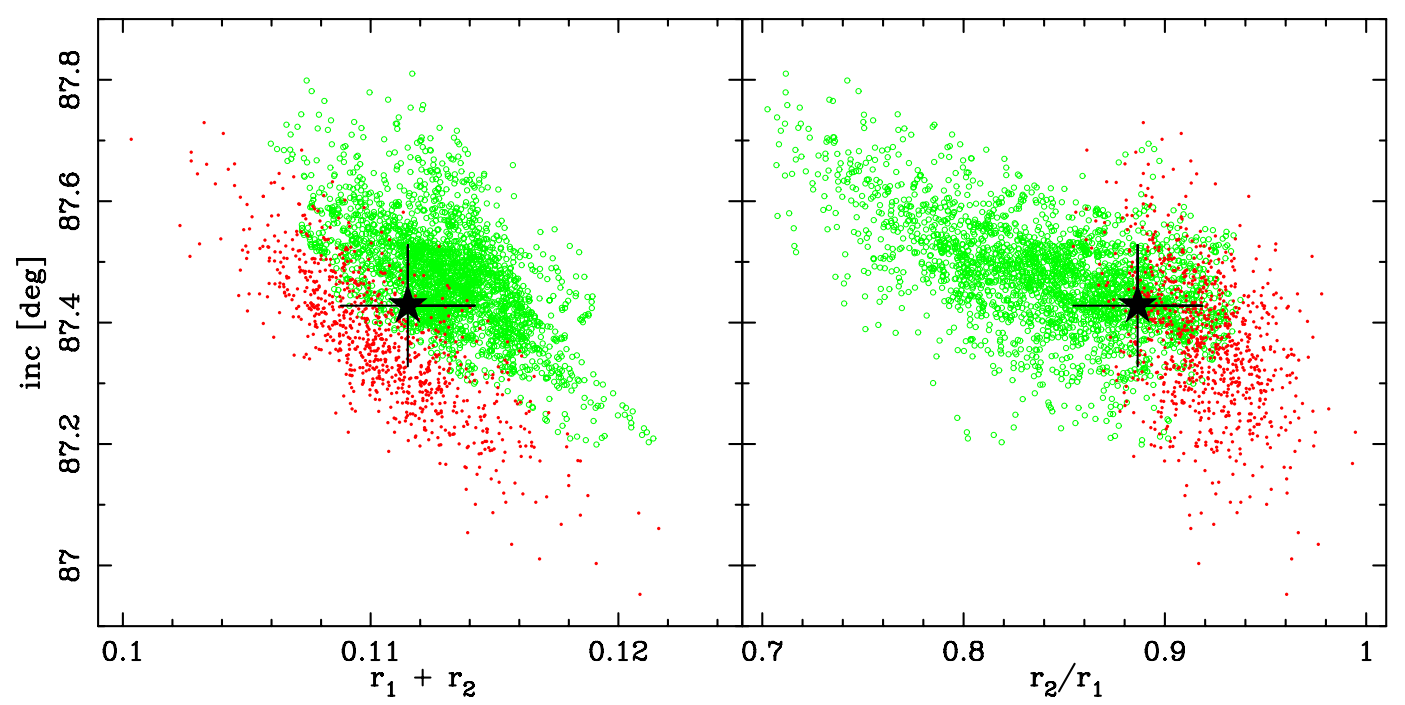

Fig. 4. Results of the residual-shifts analysis performed with JKTEBOP on the ASAS (red) and SuperWASP data (green). Plots present the distribution of consecutive solutions on the $r_{1}+r_{2}$ vs. $i$ (left) and $k=r_{2} / r_{1}$ vs. $i$ (right) panels. Black stars with error bars correspond to the adopted values with their $1 \sigma$ uncertainties.

velocities themselves are larger than predicted by the final solution and tidal locking. This could have been caused by underestimation of the assumed micro- or macro-turbulence velocity. What is surprising, is that we found two significantly different values of $[\mathrm{M} / \mathrm{H}]:-0.3$ and -0.1 for the primary and secondary, respectively, with uncertainties of 0.05 dex. We found that this difference was not dependent on the starting values of any parameter, including the flux ratio, and was not correlated with the resulting temperatures. We need to note, however, that the FEROS spectra were not corrected for the scattered light, which might have influenced the SME analysis, and that the S/N of the secondary's spectrum is significantly lower than the primary's. We thus adopt a conservative value of $[\mathrm{M} / \mathrm{H}]=-0.2 \pm 0.1 \mathrm{dex}$.

\subsection{Light curve solution}

For the light curve (LC) analysis, we used the latest version (v28) of the code JKTEBOP (Southworth et al. 2004a,b), which is based on the EBOP program (Popper \& Etzel 1981). On the basis of spectroscopic data we first found the mass ratio and ephemeris, which we included in the LC analysis. We found that the orbital period found directly by JKTEBOP from the ASAS photometry agrees with the one from RVs, however, with larger uncertainties, which leads to significantly worse orbital solution. The SuperWASP data constrain the period even worse. For JKTEBOP, we also used flux ratios found in TODCOR, and the logarithmic limb darkening (LD) law with coefficients that are interpolated from the tables of van Hamme (1996) for ASAS and Pollacco et al. (2006) for SuperWASP. The gravity darkening coefficients and bolometric albedos were always kept fixed at the values appropriate for stars with convective envelopes ( $g=0.32, A=0.5)$. As mentioned before, various tests performed on every data set did not show a significant eccentricity of the orbit of AK For; thus, $e$ was kept fixed to 0 in the analysis. We fitted for the sum of the fractional radii $r_{1}+r_{2}$, their ratio $k$, orbital inclination $i$, surface brightness ratios $J$, and brightness scales (out-of-eclipse magnitudes in each filter).

The correlated "red" noise is especially strong in the SuperWASP data (Southworth et al. 2011). Thus, to calculate reliable errors, we run task 9, which uses the residual-shifts method (Southworth 2008) to asses its importance. We have run several tests to check how the final model varies with various LD coefficients and ephemeris, but we did not notice a strong dependence. However, we let them be perturbed in the residual-shifts simulations to at least partially account for their uncertainties. Other sources of errors contribute more. It is known that the orbital inclination is correlated with the radiirelated parameters, especially their sum for systems with partial eclipses, as AK For. In Fig. 4, we show the results of the JKTEBOP analysis on the $r_{1}+r_{2}$ vs. $i$, and $k=r_{2} / r_{1}$ vs. $i$ diagrams. We see that different data sets give similar values of inclination, but different radii, nevertheless, agree with each other. The probable reason for this slight inconsistency is that we used the same flux ratio for the two different filters. However, as mentioned before, no significant change of the flux ratio was noticed in the wavelengths corresponding to the SuperWASP filter. These differences can also result from the activity and the location of spots (not included here).

As a result, we adopted weighted averages of the values found from the two data sets. We mark them in Fig. 4, with the adopted $1 \sigma$ errors. The model LCs for ASAS and SuperWASP photometry are presented in Fig. 5. The resulting values of fractional radii $r_{1,2}$, the inclination, and surface brightness ratios are given in Table 3.

The absolute values of parameters were calculated with the JKTABSDIM procedure, which is available with JKTEBOP. We assumed $E(B-V)=0.0$, the $J H K$ photometry from 2MASS (Skrutskie et al. 2006), and $[\mathrm{M} / \mathrm{H}]=0.0$ or -0.5 , which are values closest to our results of the spectral analysis. In both cases the results were practically undistinguishable. Distances were calculated with various bolometric corrections for various filters (Bessell et al. 1998; Flower 1996; Girardi et al. 2002) and surface brightness $-T_{\text {eff }}$ relations from Kervella et al. (2004). As a result we adopted their average and the standard deviation as the uncertainty. The value of $32.4 \pm 1.6 \mathrm{pc}$ agrees with the distance from HIPPARCos $(31.1 \pm 1.1 \mathrm{pc}$; van Leeuwen 2007), which proves that the flux ratio from TODCOR and the temperatures we obtained in SME are correct. The single absolute $V$ magnitudes also agree with values that are calculated on the basis of the HIPPARCOS parallax and flux ratio from TODCOR; however, the latter have smaller errors. The complete set of final parameters is presented in the Table 3. For consistency, we show distance 

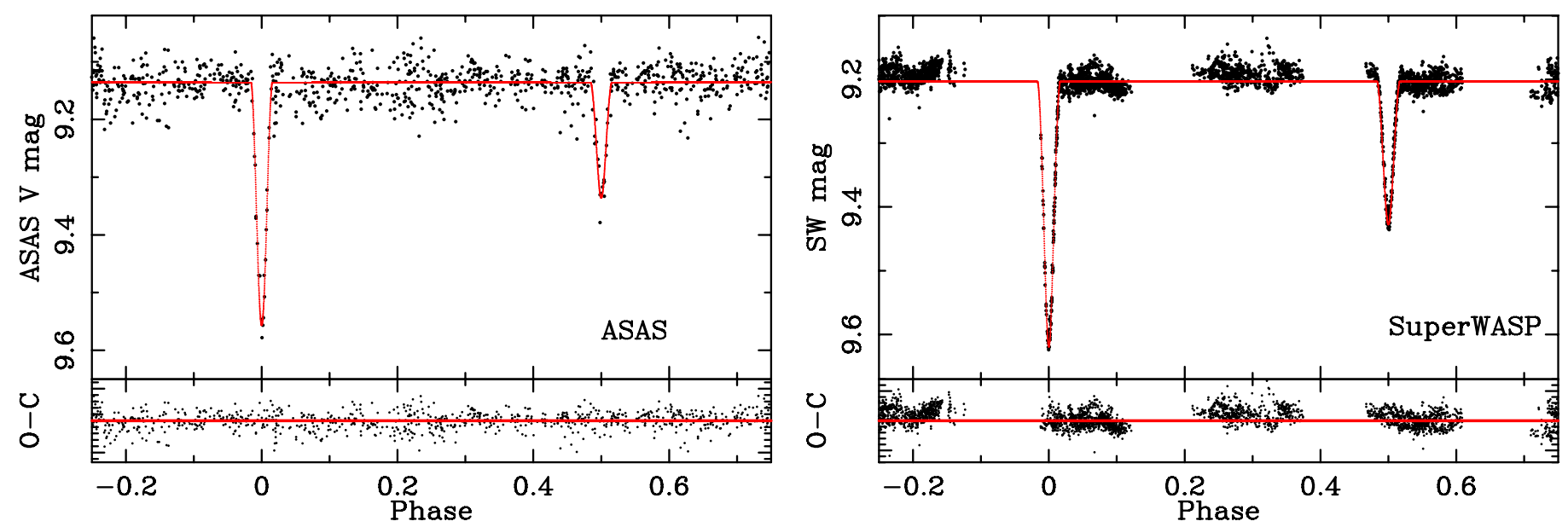

Fig. 5. Phase-folded light curves of AK For from ASAS (left) and SuperWASP (right) with the best-fitting model and residuals.

and absolute $V$ magnitudes obtained in two ways but adopt the HIPPARCos-based values as the final ones. In this way, we have a complete set of absolute physical parameters of the system not based on any models or calibrations.

\section{Discussion}

\subsection{Activity}

The activity of AK For is undoubted and was already noted (Gray et al. 2006). The system has been detected in the X-rays by the Einstein mission (Gioia et al. 1990). We found the emission in $\mathrm{H}_{\alpha}$ (Fig. 6) and in the $\mathrm{Ca} \mathrm{II} \mathrm{H}$ and $\mathrm{K}$ lines (Fig. 7; covered in our data only by HARPS). Both $\mathrm{H}_{\alpha}$ lines have an interesting double-peak profile with core absorption and emission in wings. The Ca II lines of the primary appear more prominent, but take the flux ratio of two components around $3950 \AA$ into account; it is the secondary's lines that are stronger with respect to the component's continuum. The secondary also has stronger $\mathrm{H}_{\alpha}$, but the $\mathrm{H}_{\alpha}$ emission varies in the timescale of single days for both components (Fig. 6).

The long-cadence ASAS photometry (Fig. 8) shows a clear fading trend by about 0.02 mag until JD 2453500 , which probably originates in the evolution of spots. The additional short-cadence photometry we made with PISCO (Fig. 9) also proves the presence of spots, causing brightness variations of a similar scale, as the long-term trend seen in the ASAS data. It is impossible, however, to constrain the spot pattern, or even to point out the component on which they are present (most likely on both). If AK For's brightness is still at the level from September 2009 (the end of ASAS data), both components are probably substantially and uniformly covered with spots. Additional, continuous, high-precision photometry from a global telescope network is required to constrain the pattern and location of spots in a given time. With such data, it would also be possible to significantly improve the precision in radii and make AK For one of the best-studied eclipsing systems.

\subsection{Kinematics}

The main sequence evolutionary stage of AK For is suggested by its galactic kinematics. We used our determinations of the systemic velocity with the position, proper motion, and distance from HIPPARCos (Table 1). The obtained values of $U=-28 \pm$ $1 \mathrm{~km} \mathrm{~s}^{-1}, V=-12.8 \pm 0.8 \mathrm{~km} \mathrm{~s}^{-1}$ and $W=18.2 \pm 0.7 \mathrm{~km} \mathrm{~s}^{-1}$ (no correction for the Solar motion has been made), put marginally AK For in the galactic thin disk (Seabroke \& Gilmore 2007). This suggests the age below 4.5-5 Gyr; however, older stars are observed in the thin disk (Allende Prieto 2010, and references therein). Taking the large value of $W$ into account at the edge of the thin disk distribution, a young age (below $1 \mathrm{Gyr}$ ) seems to be unlikely.

\subsection{Comparison with theoretical models}

We reached a very high precision of $0.14+0.11 \%$ in the masses and also a good precision in radii $(2.9+2.7 \%)$, which allows for meaningful tests of the stellar evolution models. The AK For has the most precise mass measurements among low-mass eclipsing binaries, and it is the brightest one $(V=9.135 \mathrm{mag}$ from the ASAS light curve). It is one of the most accurately measured eclipsing binaries in general. The precision in masses was possible due to relatively low rotational velocities, as related to the period that is longer than for the majority of similar DEBs. Improving the accuracy of the radii and related radiative parameters requires more precise and better-calibrated photometry.

In Fig. 10, we compare our measurements with the Dartmouth stellar evolution models (Dotter et al. 2007) in the mass $M$ vs. $R, \log \left(T_{\text {eff }}\right), \log \left(L / L_{\odot}\right)$ and $M_{V}$ planes. We show 1 and $6 \mathrm{Gyr}$ isochrones for three values of $[\mathrm{Fe} / \mathrm{H}]$ spanning from -0.1 to $-0.3 \mathrm{dex}^{4}$.

One can see that the $6 \mathrm{Gyr}$ isochrone for the $[\mathrm{Fe} / \mathrm{H}]$ value, as obtained in the SME analysis, fits to all the parameters within $1 \sigma$. The system still resides on the main sequence but has evolved and the 1 Gyr models clearly do not fit the data. This shows how useful the spectral analysis is in solving the age-metallicity degeneracy on the main sequence. The age of 6 Gyr was found to be the best-fitting one, especially on the $M-R$ plane. It agrees with the kinematic analysis described in the previous section.

\subsection{4-0.9 $M_{\odot}$ stars in detached eclipsing binaries (DEBs)}

The AK Fornacis is unique system for several reasons. First, it is bright and close, allowing for further detailed studies, which may, however, be problematic due to the unfortunate orbital period. Second, its component masses fall in the relatively poorly

\footnotetext{
4 SME gives $[\mathrm{M} / \mathrm{H}]$ but the transformation to $[\mathrm{Fe} / \mathrm{H}]$ gives $-0.194_{-0.099}^{+0.097}$ in case of the Dartmouth set; thus, we adopted $[\mathrm{Fe} / \mathrm{H}]=-0.2 \pm 0.1$.
} 
K. G. Hełminiak et al.: Orbital and physical parameters of eclipsing binaries from the ASAS catalogue. VI.

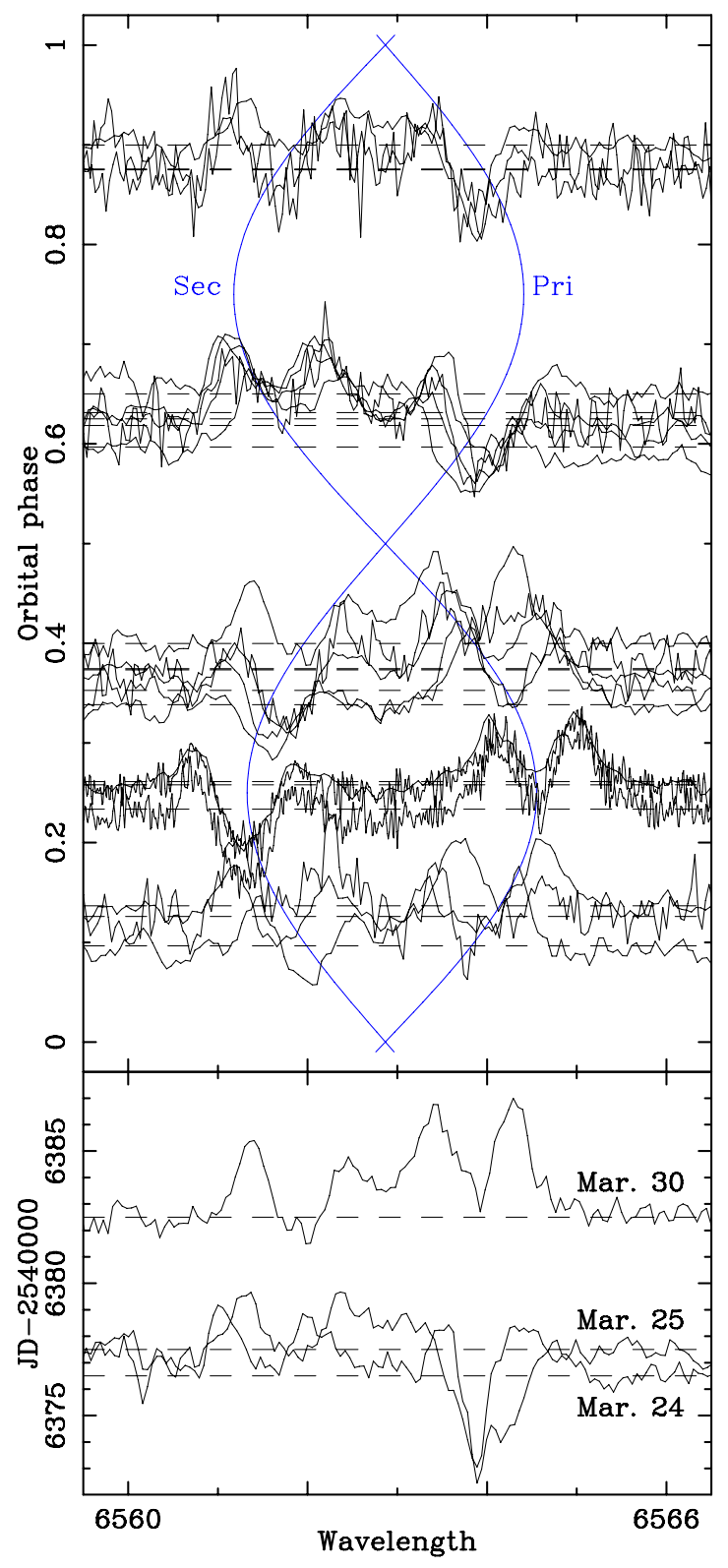

Fig. 6. $\mathrm{H}_{\alpha}$ emission lines of AK For. In a given panel, all spectra were continuum-fitted and equally scaled. The dashed lines show the level of continuum and mark the orbital phase or time of the observation. Top: all spectra are shown as a function of the orbital phase. Solid blue lines trace the position of the $\mathrm{H}_{\alpha}$ line as predicted by the orbital solution. Bottom: three FEROS spectra from March 2013, which show rapid evolution of the $\mathrm{H}_{\alpha}$ profiles. On March 30, the emission was much stronger than a few days earlier. Secondary's emission is the stronger one.

studied range of $0.5-0.8 M_{\odot}$, between usually very active and mostly convective M-dwarfs and usually inactive solar analogues of the $\mathrm{G}$ type. The activity is thought to be the major factor responsible for the observed discrepancies between measurements and theory in binaries, which tend to be larger for later spectral types. It is notable that the theory predicts the measurements reasonably well in this case. Finally, the orbital period of AK For is longer than for the majority of well-measured DEBs with $\mathrm{K}, \mathrm{M}$, and late-G type components.

In the online DEBCat catalogue ${ }^{5}$ of well measured eclipsing binaries, there are almost no stars of masses lower than $0.7 M_{\odot}$

\footnotetext{
5 http://www.astro.keele.ac.uk/ jkt/debcat/
}

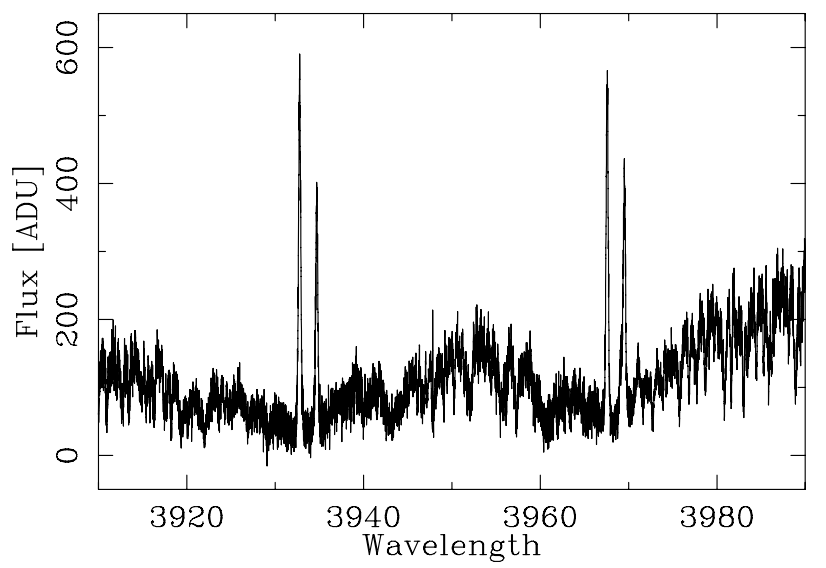

Fig. 7. Portion of HARPS spectrum of AK For around $\mathrm{Ca}$ II $\mathrm{H}$ and $\mathrm{K}$ lines. Emission from both components in the cores is clearly visible. The apparently stronger lines come from the primary but due to the fainter continuum of the secondary star, its emission lines are intrinsically stronger. Spectrum taken in quadrature.

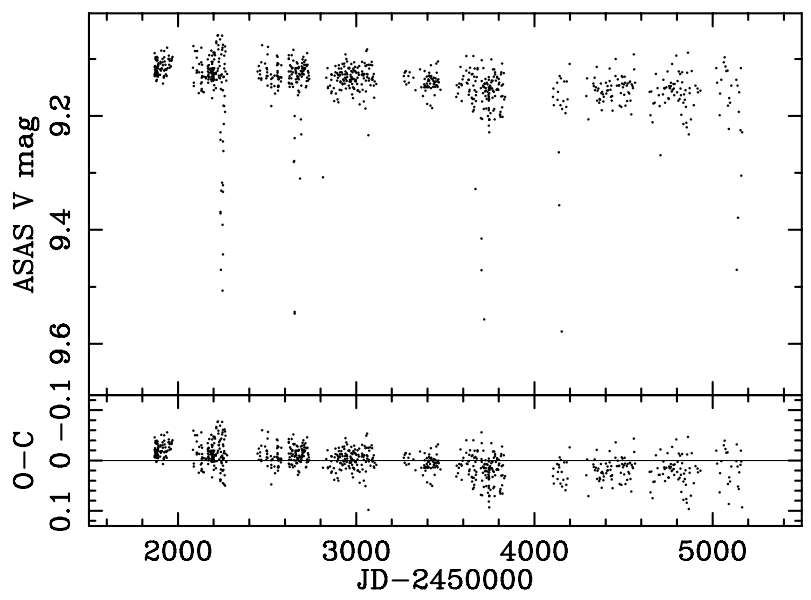

Fig. 8. Long cadence ASAS light curve (top) and the residuals of the best-fitting model from the Fig. 5 (bottom) as a function of time. The change of the total brightness of the system is clearly visible, and can be explained by the evolution of the spot pattern.

for periods longer than three days. The notable exceptions are the secondary of M 55-V $54\left(0.56 M_{\odot}, 9.27\right.$ d; Kałużny et al. 2014), the secondary of KIC $6131659\left(0.69 M_{\odot}, 17.53 \mathrm{~d}\right.$; Bass et al. 2012), both components of LSPM J1112+7626 (much lower masses $0.39+0.27 M_{\odot}, 41.03 \mathrm{~d}$; Irwin et al. 2011), or both components of T-Lyr1-17236 $\left(0.68+0.53 M_{\odot}, 8.43 \mathrm{~d}\right.$; Devor et al. 2008 , not included due to poor precision in radii). It is obviously an observational selection effect, and it is desirable to discover more long-period ( $>3 \mathrm{~d}$ ) low-mass eclipsing binaries.

Figure 11 shows the mass-radius diagram for the wellmeasured eclipsing binaries with at least one component in the $0.4-0.9 M_{\odot}$ mass range. We show systems from the DEBCat, including three examples from our previous research (Hełminiak \& Konacki 2011; Hełminiak et al. 2011). Dartmouth isochrones for $1,5,10$, and $14 \mathrm{Gyr}$ and two metallicities are plotted over. It is clearly seen that age and metallicity plays an important role in the $M-R$ distribution, at least for stars more massive than $0.6 M_{\odot}$. Traditionally, the radii and temperatures of low mass stars were compared to the $1 \mathrm{Gyr}$ solar-composition isochrone. The general characteristic was that the observed radii are larger and effective temperatures lower than models predicted. We now know a number of late-type systems old enough 


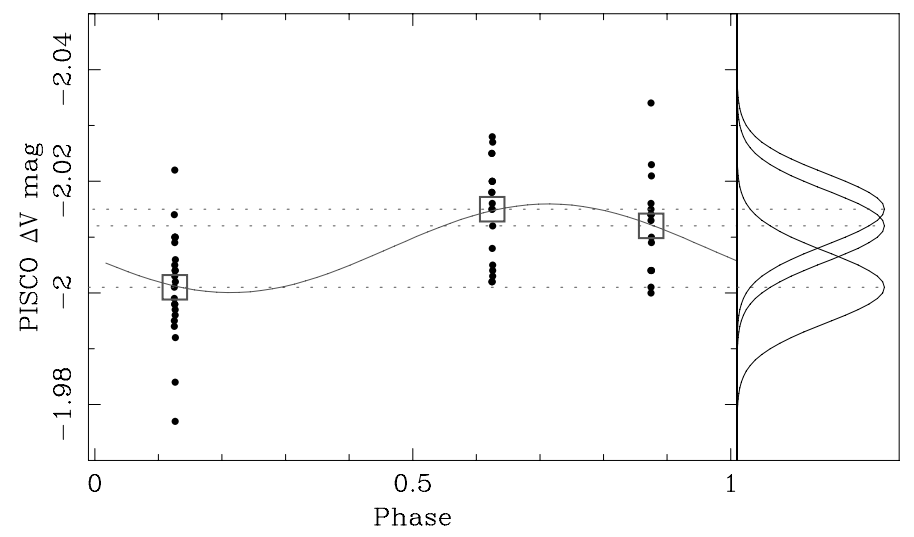

Fig. 9. PISCO $V$-band photometry of AK For, when taken simultaneously with CORALIE observations in February 2013, which is phasefolded with the orbital period. For each night, squares mark the average values, their error is $2 \mathrm{mmag}$ (size of symbols), and the dispersion is 9 mmag; corresponding Gaussians are shown to the right. The presence of spots can be deduced from a small but clear fading around phase 0.126 , when the spot is presumably well visible. The brightness drop is 0.011 mag relative to the previous night (phase 0.875 ). The solid line is sine-fitted to data, plotted to guide the eye only, and has no physical meaning.

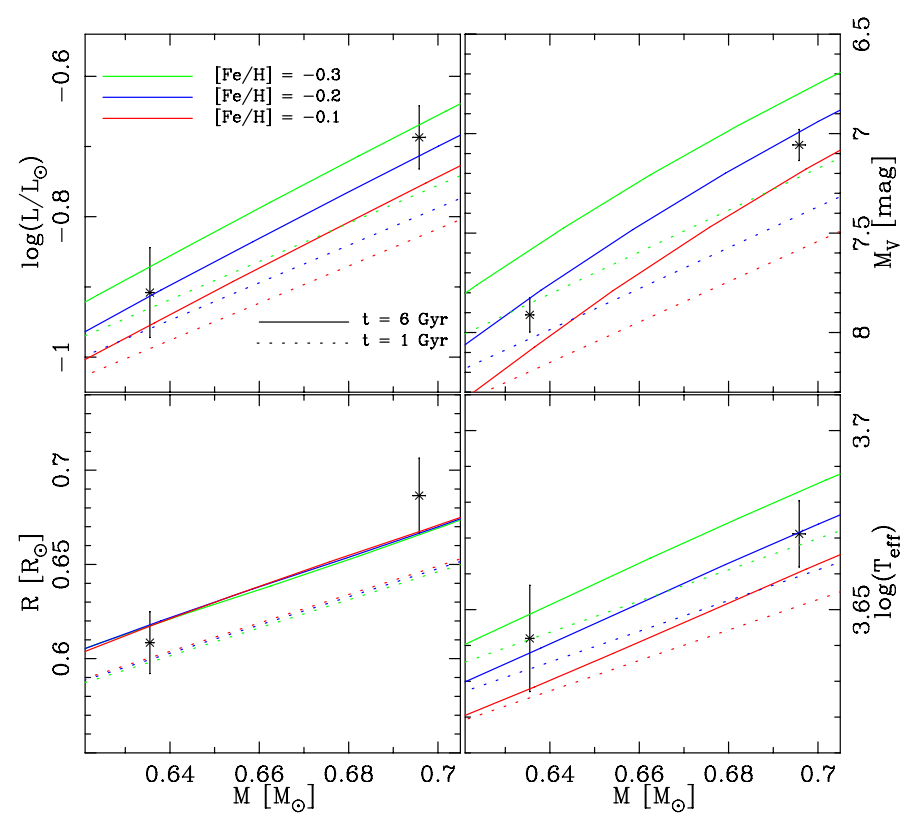

Fig. 10. Comparison of our results with the Dartmouth stellar evolution models for three values of $[\mathrm{Fe} / \mathrm{H}]$ and ages of 1 and $6 \mathrm{Gyr}$.

to have their radii enlarged by the evolution (AK For should be considered one of them). In some cases, metallicity can also be estimated from the spectra, which helps to constrain the age. As one can see, the traditional approach is not always valid. To indicate if a given star's radius is enlarged or not, one needs an independent metallicity and age estimation (possible only in clusters), or one may check if the slope that the two components define on the $M-R$ diagram is compatible with any normal evolutionary relation. Usually, the secondary appears to be oversized (over-aged), but literature examples show that it may also be the primary.

According to the recently popular explanation of the observed oversized radii of low-mass DEBs, the high rotational velocity, which results from a short orbital period, is responsible

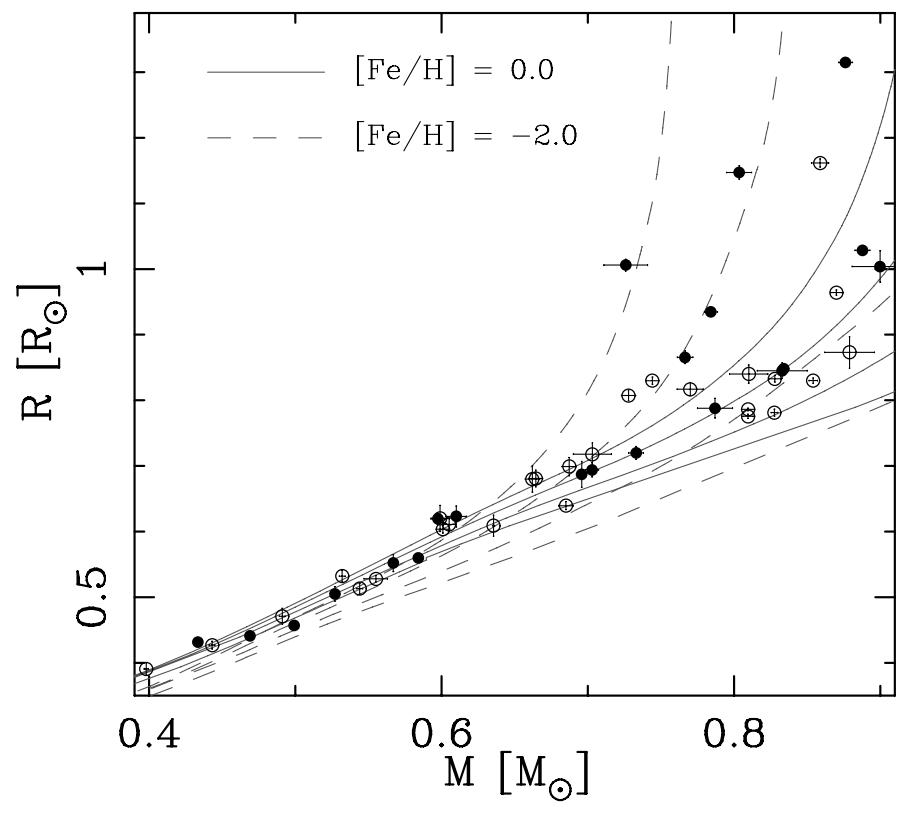

Fig. 11. Mass-radius diagram for well-measured DEBs with at least one component in the mass range of $0.4-0.9 M_{\odot}$. Solid symbols represent primary (more massive) and open symbols secondary components. Dartmouth isochrones of solar composition (solid) and metal-depletion (dashed) are plotted for ages 1, 5, 10 and 14 Gyr.

for a higher level of activity, which inhibits the effectiveness of convection. This leads to larger radii and lower temperatures (Chabrier et al. 2007). Long-period systems should, thus, be less active and have their radii and temperatures better reproduced than the short-period binaries. One can see in Fig. 10 that AK For does not follow this trend, despite being active. On the other hand, the $0.85 M_{\odot}$ secondary of a 4.8 d system V636 Cen (Clausen et al. 2009), and both components of the $41 \mathrm{~d}$ period LSPM J1112+7626 (Irwin et al. 2011) are both active and inflated. It is worth mentioning that the Ca II emission lines are much stronger in AK For than in V636 Cen, which rotates a bit faster. Some of the components of longer-period systems found in globular clusters, like the secondaries of V66 and V69 in M4 (Kałużny et al. 2013) or the primary of V54 in M55 (Kałużny et al. 2014), also seem to be oversized, but their activity is not seen. In the same time, we also know short-period active systems, like ASAS J045304-0700.4 $(P=1.77 \mathrm{~d}$; Hełminiak \& Konacki 2011), MG1-78457 ( $P=1.59$ d), MG1-506664 $(P=1.55 \mathrm{~d}$; Kraus et al. 2011), or KOI-126 BC $(P=1.77 \mathrm{~d}$; Feiden et al. 2011), where the model radii fit the observed ones. In light of recent findings, it is difficult to draw any conclusions about the connection between rotation, activity, radii, and effective temperatures.

\section{Summary}

We present the first full orbital and physical analysis of the brightest low-mass detached eclipsing binary known to date AK Fornacis. The system's characteristics (brightness and long orbital period) allow for accurate determination of masses and radii. The precision in masses is very high but still somewhat limited by the stellar activity. The object's characteristics are unusual among the known low-mass DEBs. Despite the activity, the properties of both components are nicely reproduced and the age can be determined fairly well as for a main sequence system. The reason for the enhanced activity cannot be the rotation, 
K. G. Hełminiak et al.: Orbital and physical parameters of eclipsing binaries from the ASAS catalogue. VI.

as AK For rotates rather slowly, although it is faster than typical, single field $\mathrm{K}$ and $\mathrm{M}$ dwarfs.

The major observational disadvantage of the system is its period, which is very close to $4 \mathrm{~d}$ and makes the eclipses not always possible to observe from one place during a single season. Further work on this system requires continuous photometry from a global network of telescopes, such as WET (Nather et al. 1990), Solaris (Konacki et al. 2012), or HAT-S (Bakos et al. 2009), to derive even more accurately crucial parameters, like the radii or temperatures.

Acknowledgements. We would like to thank Dr Johannnes Andersen for the discussion and valuable comments that helped us improve our manuscript, and the staff of the La Silla and Geneva observatories, especially G. Lambert and D. Naef, for their hospitality and help during the observations. K.G.H. acknowledges support provided by the Proyecto FONDECYT Postdoctoral No. 3120153, the Polish National Science Center grant 2011/03/N/ST9/01819, and the National Astronomical Observatory of Japan as Subaru Astronomical Research Fellow. R.B. and N.E. are supported by CONICYT-PCHA/Doctorado Nacional. M. Ratajczak is supported by the Polish National Science Center through grant 2011/01/N/ST9/02209. A.J. acknowledges support from FONDECYT project 1130857, BASAL CATA PFB-06, and grant IC120009 awarded to the Millennium Institute of Astrophysics, MAS, by the Millennium Science Initiative of the Chilean Ministry of Economy, Development, and Tourism. M.K. is supported by the European Research Council Starting Grant, the Polish National Science Center through grant 5813/B/H03/2011/40, the Ministry of Science and Higher Education through grant W103/ERC/2011 and the Foundation for Polish Science through grant "Ideas for Poland". M. Rabus acknowledges support from FONDECYT postdoctoral fellowship No. 3120097. We have used data from the WASP public archive in this research. The WASP consortium comprises of the University of Cambridge, Keele University, University of Leicester, The Open University, The Queen's University Belfast, St. Andrews University and the Isaac Newton Group. Funding for WASP comes from the consortium universities and from the UK's Science and Technology Facilities Council. This publication makes use of data products from the Two Micron All Sky Survey, which is a joint project of the University of Massachusetts and the Infrared Processing and Analysis Center/California Institute of Technology, funded by the National Aeronautics and Space Administration and the National Science Foundation.

\section{References}

Allende Prieto, C. 2010, Proc. IAU Symp., 265, 304

Bailer-Jones, C. A. L. 2011, MNRAS, 411, 435

Bakos, G. Á., Noyes, R., Kovács, G., et al. 2009, Proc. IAU Symp., 253, 21

Bass, G., Orosz, J., Welsh, W. F., et al. 2012, ApJ, 761, 157

Bessell, M. S., Castelli, F., \& Plez, B. 1998, A\&A, 333, 231

Chabrier, G., Gallardo, J., \& Baraffe, I. 2007, A\&A, 427, 17

Clausen. J. V., Bruntt, H., Claret, A., et al. 2009, A\&A, 502, 523

Demory, N.-O., Gillon, M., Deming, D., et al. 2011, A\&A, 533, A114

Devor, J., Charbonneau, D., Torres, G., et al. 2008, ApJ, 687, 1253

Dotter, A., Chaboyer, B., Jevremović, D., et al. 2007, AJ, 134, 376
Feiden, G. A., Chaboyer, B., \& Dotter, A. 2011, ApJ, 740, 25

Flower, P. J. 1996, ApJ, 469, 335

Giota, M., Maccacaro, T., Schild, R. E., et al. 1990, ApJS, 72, 567

Girardi, L., Bertelli, G., Bressan, A., et al. 2002, A\&A, 391, 195

Gray, R. O., Corbally, C., J., Garrison, R. F., et al. 2006, AJ, 132, 161

Hełminiak, K. G., \& Konacki, M. 2011, A\&A, 526, A29

Hełminiak, K. G., Konacki, M., Złoczewski, K., et al. 2011, A\&A, 527, A14

Holman, M. J., \& Wiegert, P. A. 1999, AJ, 117, 621

Irwin, J. M., Quinn, S. N., Berta, Z. K., et al. 2011, ApJ, 742, 123

Jordán, A., Brahm, R., \& Bakos, G. Á. 2014, AJ, 148, 29

Kałużny, J., Thompson, I. B., Różyczka, M., et al. 2013, AJ, 145, 43

Kałużny, J., Thompson, I. B., Dotter, A., et al. 2014, Acta Astron., 64, 11

Kaufer, A., Stahl, O., Tubbesing, S., et al. 1999, The Messenger, 95, 8

Kazarovets, E. V., Samus, N. N., Durlevich, O. V., et al. 1999, IBVS, 4659

Kervella, P., Thévenin, F., Di Folco, E., \& Ségransan, D. 2004, A\&A, 426, 297

Konacki, M., Muterspaugh, M. W., Kulkarni, S. R., \& Hełminiak, K. G. 2010, ApJ, 719, 1293

Konacki, M., Sybilski, P., Kozłowski, S. K., Ratajczak, M., \& Hełminiak, K. G. 2012, Proc. IAU Symp., 282, 111

Kraus, A. L., Tucker, R. A., Thompson, M. I., Craine, E. R., \& Hillenbrand, A. 2011, ApJ, 728, 48

Kupka, F., Piskunov, N., Ryabchikova, T. A., Stempels, H. C., \& Weiss, W. W. 1999, A\&AS, 138, 119

Kurucz, R. L. 1993, VizieR Online Data Catalog: VI/39

Lacy, C. H. 1977, ApJ, 218, 444

Mayor, M., Pepe, F., Queloz, D., et al. 2003, The Messenger, 114, 20

Mermilliod, J.-C. 1986, Catalogue of Eggen's UBV data

Morales, J. C., Ribas, I., Jordi, C., et al. 2009, ApJ, 691, 1400

Nather, R. E., Winget, D. E., Clemens, J. C., et al. 1996, ApJ, 361, 309

Otero, S. A. 2003, IBVS, 5480, 1

Piskunov, N. E., Kupka, F., Ryabchikova, T. A., Weiss, W. W., \& Jeffery, C. S. 1995, A\&AS, 112, 525

Pojmański, G. 2002, Acta Astron., 52, 397

Pollacco, D. L., Skillen, I., Collier Cameron, A., et al. 2006, PASP, 118, 1407

Popper, D. M. 1997, AJ, 114, 1195

Popper, D. M., \& Etzel, P. B. 1981, AJ, 86, 102

Seavroke, G. M., \& Gilmore, G. 2007, MNRAS, 380, 1348

Skrutskie, M. F., Cutri, R. M., Steiening, R., et al. 2006, AJ, 131, 1163

Southworth, J. 2008, MNRAS, 386, 1644

Southworth, J., Maxted, P. F. L., \& Smalley, B. 2004a, MNRAS, 351, 1277

Southworth, J., Zucker, S., Maxted, P. F. L., \& Smalley, B. 2004b, MNRAS, 355 986

Southworth, J., Pavlovski, K., Tamajo, E., et al. 2011, MNRAS, 414, 3740

Thompson, I. B., Kałużny, J., Rucinski, S. M., et al. 2010, AJ, 139, 329

Torres, G., \& Ribas, I. 2002, ApJ, 567, 1140

Torres, G., Andersen, J., \& Giménez, A. 2010, A\&ARv, 18, 67

Upgren, A. R., Grossenbacher, R., Penhallow, W. S., MacConnell, D. J., \& Frye, R. L. 1972, AJ, 77, 486

Valenti, J. A., \& Fischer, D. A. 2005, ApJS, 159, 141

Valenti, J. A., \& Piskunov, N. 1996, A\&AS, 118, 595

van Hamme, W. 1996, AJ, 106, 2096

van Leeuwen, F. 2007, A\&A, 474, 653

Worthey, G., \& Lee, H.-C. 2011, ApJS, 193, 1

Zucker, S., \& Mazeh, T. 1994, ApJ, 420, 806 